\section{$\underset{\substack{\text { hommes } \\ \text { \& migrations }}}{ }$}

\section{Hommes \& migrations}

Revue française de référence sur les dynamiques

migratoires

1309 | 2015

Le $3 e$ âge des migrants

\title{
L'offre de soins de santé aux Français en Chine
}

\section{Marc Antoine}

\section{(2) OpenEdition \\ Journals}

\section{Édition électronique}

URL : http://journals.openedition.org/hommesmigrations/3115

DOI : 10.4000/hommesmigrations.3115

ISSN : 2262-3353

\section{Éditeur}

Musée national de l'histoire de l'immigration

\section{Édition imprimée}

Date de publication : 1 janvier 2015

Pagination : 182-187

ISBN : 978-2-919040-30-8

ISSN : $1142-852 X$

\section{Référence électronique}

Marc Antoine, "L'offre de soins de santé aux Français en Chine », Hommes \& migrations [En ligne], 1309 | 2015, mis en ligne le 17 juillet 2015, consulté le 24 septembre 2020. URL : http:// journals.openedition.org/hommesmigrations/3115; DOl : https://doi.org/10.4000/ hommesmigrations.3115 


\section{REPÉRAGE}

\section{L'OFFRE DE SOINS DE SANTÉ AUX FRANÇAIS EN CHINE}

MARC ANTOINE, médecin-chef de l'ambassade de France en Chine

$\mathrm{L}^{2}$ a communauté française en Chine connaît une évolution par la venue de Français sur emplois précaires ou à revenu intermédiaire. Il existe une inégalité dans l'accès aux soins de santé chez les Français résidant en Chine en fonction de leur statut socio-économique. Le système de santé en Chine ne satisfait pas pleinement la communauté française.

La santé est un problème quotidien des Français résidant en Chine. Cet article se propose, à partir d'une enquête de type sociologique, d'évaluer l'offre de soins aux Français résidant ou de passage à Pékin. Entre le $1^{\text {er }}$ octobre 2013 et fin juin 2014, nous avons fait passer un questionnaire écrit en langue française, comportant à la fois des questions ouvertes et fermées, à tous les Français majeurs ou à leur conjoint de nationalité étrangère consultant au cabinet médical de l'ambassade de France en Chine. $100 \%$ des personnes ont accepté de répondre au questionnaire. Au 31 décembre 2013, le nombre d'inscrits à Pékin sur le registre de l'ambassade de France' était de 4790 personnes. 304 personnes ont répondu à cette enquête, soit environ $9 \%$ des personnes majeures inscrites sur ce registre. Pour l'analyse, nous avons procédé à un premier tri à plat puis à des tris croisés. Notre enquête comptabilise $52 \%$ d'hommes et $48 \%$ de femmes. C'est une population jeune, souvent venue en famille. En effet, $65 \%$ des personnes majeures ont entre 18 et 40 ans et
$31 \%$ entre 41 et 60 ans. 41 \% des Français résidant en Chine déclarent vivre avec leurs enfants.

Le statut professionnel et les assurances santé sont des indicateurs essentiels pour mettre en évidence les inégalités d'accès aux soins de santé des Français résidant en Chine et pour mieux comprendre le niveau de satisfaction des soins qu'ils ont reçus. Le statut professionnel est une information utile pour connaître le niveau socioéconomique d'une personne. Chez les Français résidant en Chine, on différencie ceux qui ont des contrats locaux avec des salaires souvent faibles, soumis à la législation locale (c'està-dire en Chine onze jours de congés payés par an et une protection sociale de santé chinoise) et ceux qui bénéficient de contrats expatriés avec un salaire très élevé et de multiples avantages (billets d'avion pour la France, logement, voiture de fonction avec chauffeur, assurance santé internationale et assistance rapatriement, scolarité des enfants dans des écoles internationales).

Dans notre enquête, on note une majorité de contrats expatriés (44\%) contre $19 \%$ de contrats locaux. Enfin, nous avons $15 \%$ d'étudiants, $8 \%$ d'entrepreneurs, $7 \%$ de volontaires internationaux, $4 \%$ de stagiaires et $2 \%$ de sans emploi. $5 \%$ des patients sont de passage en Chine pour une durée de moins de trois mois. Dans l'enquête de Shanghai de $2012^{2}$, on trouve une majorité de contrats locaux ( $55 \%$ ), cela repré- 
sente presque deux fois les contrats expatriés (30\%), alors qu'en 2008 les contrats expatriés étaient supérieurs aux contrats locaux (47\% et $40 \%$ respectivement). Ces chiffres montrent un "accroissement de Français sur emplois précaires ou à revenu intermédiaire". Cela a des conséquences notamment sur l'accès à la santé et à des soins de qualité. Car ces Français n'ont pas les moyens de payer une assurance santé ou bien ils adhèrent à une assurance santé bon marché qui ne rembourse pas l'intégralité des frais de santé. Pourquoi avons-nous une majorité de contrats expatriés dans notre enquête ? D'une part, les familles françaises en contrat expatrié sont mieux connues des services de l'ambassade de France. Ainsi, elles ont accès plus facilement à ses informations. En 2013, 29 \% des Français ayant consulté le médecin de l'ambassade de France sont les familles des agents de l'ambassade. D'autre part, à cause de leurs difficultés financières, les Français en situation de précarité retardent leur consultation chez un médecin en cas de problème de santé, voire consultent à l'hôpital public chinois, ce qui est moins cher que de consulter un médecin français.

\section{La précarité de la communauté française de Chine}

En Chine, la réforme de la protection sociale de

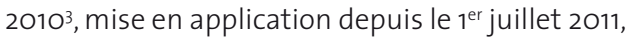
permet à chaque étranger résidant et travaillant légalement en Chine d'avoir obligatoirement une couverture sociale de santé. Cependant, une liste de trois hôpitaux publics chinois pour se faire soigner est imposée pour être remboursé, les cliniques internationales privées et le cabinet médical de l'ambassade de France n'en faisant pas par- tie. Les taux de remboursement dépendent des cotisations versées, c'est-à-dire du contrat de travail. Toutes les pathologies ne sont pas remboursées, notamment les cancers. Le choix du médecin est libre. II n'y a pas de médecin référent et I'on peut consulter directement n'importe quel spécialiste. Les consultations VIP4 dans les hôpitaux publics chinois, proposant une amélioration des services liés à l'hôtellerie, ne sont pas non plus remboursées. Le ticket modérateur est à la charge de l'assuré. En cas d'hospitalisation, les frais remboursés restent limités à 12000 yuans (RMB) par an maximum, soit moins de $1500 €$ Dans ces conditions, la plupart des Français choisissent de souscrire à une autre assurance santé. Dans notre enquête, 86 \% des personnes ont une assistance rapatriement, $94 \%$ ont une assurance santé et $57 \%$ ont une assurance santé complémentaire.

Parmi les assurances santé souscrites, on retrouve notamment $25 \%$ d'adhésions à la Caisse des Français de l'étranger (CFE) $)^{5}, 14 \%$ à April International, $13 \%$ à la Mutuelle générale de l'Éducation nationale (MGEN) et $9 \%$ de détachés de la Sécurité sociale française. Toutes les autres assurances sont privées. Au total, la part des assurances santé privées représente $66 \%$. Parmi les assurés, 31 \% déclarent être remboursés en totalité dans les cliniques internationales privées, $27 \%$ ne sont pas remboursés en totalité et $42 \%$ ne savent pas le taux de leur remboursement.

Ce faible pourcentage de personnes totalement remboursées met en évidence des difficultés d'accès aux soins de santé, puisque les frais médicaux dans les cliniques internationales privées en Chine sont extrêmement onéreux. Pour prendre un exemple, la CFE ne rembourse que $70 \%$ du prix des consultations sur la base du

2. Consulat de France à Shanghai, "Résultats du sondage sur les besoins et attentes de la communauté française de Shanghai", 2012. Consultable sur: http://www.ambafrance-cn.org 3. Marc Antoine, "Système de santé et accès aux soins publics en Chine", in Conférence, "La sécurité sociale en Chine", Association des familles franco-chinoise de Pékin/ambassade de France, 3 juin 2013. 4. Very important person. 5. La CFE est une assurance pour les Français de l'étranger qui fonctionne comme la Sécurité sociale française. 


\section{REPÉRAGE}

\section{/// Tableau 1. Niveau de satisfaction des Français à l'égard des soins reçus en Chine.}

\begin{tabular}{|l|c|c|c|c|}
\hline & Hospitalisation & $\begin{array}{c}\text { Consultation dans } \\
\text { un hôpital public chinois } \\
\text { (consultation VIP } \\
\text { ou ordinaire) }\end{array}$ & $\begin{array}{c}\text { Consultation } \\
\text { dans une clinique } \\
\text { internationale } \\
\text { privée }\end{array}$ & $\begin{array}{c}\text { Système } \\
\text { de santé en } \\
\text { Chine }\end{array}$ \\
\hline Très satisfait & $45 \%$ & $17 \%$ & $45 \%$ & $11 \%$ \\
\hline Assez satisfait & $37 \%$ & $47 \%$ & $42 \%$ & $59 \%$ \\
\hline Peu satisfait & $12 \%$ & $26 \%$ & $10 \%$ & $24 \%$ \\
\hline $\begin{array}{l}\text { Pas du tout } \\
\text { satisfait }\end{array}$ & $6 \%$ & $10 \%$ & $3 \%$ & $6 \%$ \\
\hline
\end{tabular}

Source : enquête personnelle, 2014. Échantillon de 304 personnes.

tarif appliqué en France, soit 16,10 €.

Si l'adhérent n'a pas souscrit à une assurance santé complémentaire, le reste des frais médicaux sera à sa charge. L'enquête révèle que seulement $56 \%$ des personnes ayant la CFE ont une assurance santé complémentaire.

Ces résultats confirment l'évolution vers une précarité grandissante de la communauté française de Chine. Le prix moyen d'une consultation dans un établissement international privé à Pékin est de 100 à $150 €$, celui d'une consultation VIP dans un hôpital public chinois est de 25 à $35 €$, le tarif d'une consultation au cabinet médical de l'ambassade de France est de 20 à $30 €$, alors qu'une consultation ordinaire à l'hôpital public chinois est de $1 €$. Par conséquent, les Français qui n'ont pas une bonne assurance santé retardent leur prise en charge médicale ou préfèrent ne pas du tout consulter, y compris à l'hôpital public chinois pourtant peu onéreux.

\section{Une inégalité dans l'accès aux soins}

Il faut rappeler qu'environ la moitié des personnes interrogées dans notre enquête ont un contrat expatrié. Cela influence les résultats puisque ces Français ont des moyens financiers suffisants pour payer les soins de meilleure qualité existant en Chine. Le taux de satisfaction est donc très certainement surestimé.

L'enquête met en évidence un taux de satisfaction très élevé pour les hospitalisations. Notre questionnaire nous révèle que $80 \%$ des personnes étaient hospitalisées dans des établissements privés contre $20 \%$ dans des établissements publics. Le service de l'hôtellerie est d'excellente qualité dans les établissements privés et satisfait très largement la population qui peut se permettre de s'offrir ces services exorbitants, d'un coût plus 
élevé que les frais d'une hospitalisation en France, tant en public qu'en privé. De même, on comprend mieux pourquoi la satisfaction est plus élevée chez les personnes qui consultent dans les cliniques internationales privées que dans les hôpitaux publics chinois. Ces cliniques internationales privées engagent des médecins étrangers, ce qui permet de fournir une qualité des soins avec des standards internationaux. Cependant, il est important de préciser que la plupart des médecins spécialistes sont des médecins chinois qui n'ont pas reçu la même formation médicale que les médecins occidentaux.

Les résultats nous montrent qu'environ deux tiers des personnes sont satisfaites du système de santé en Chine (très ou assez satisfaites), en incluant les établissements de santé publics et privés et en comparant la qualité des soins par rapport au coût financier, contre un tiers non satisfaites (peu ou pas du tout satisfaites). Pouvons-nous prouver que le statut professionnel, voire le fait d'avoir une assurance santé ou non, c'est-à-dire le statut socio-économique d'un individu, influence le niveau de satisfaction des soins reçus en Chine ? Nous avons croisé les résultats de la satisfaction du système de santé en Chine avec le statut professionnel, puis avec l'assurance santé.

Si l'on regroupe les contrats expatriés, les contrats locaux avec un salaire supérieur à $4000 €$ net par mois (soit un quart des contrats locaux) et les volontaires internationaux (qui ont une excellente assurance santé incluse dans leur contrat de travail), $75 \%$ des personnes interrogées sont satisfaites du système de santé en Chine contre $25 \%$ non satisfaites, alors que le groupe des stagiaires, des étudiants, des contrats locaux avec un salaire inférieur à $4000 €$ nets par mois et des sans-emploi comptabilise $63 \%$ de personnes satisfaites contre $37 \%$ de non satisfaites.

Parmi les personnes qui ont une assurance santé, $73 \%$ sont satisfaites du système de santé en
Chine, alors que lorsqu'elles n'ont pas d'assurance santé, $38 \%$ le sont seulement.

D'après ces résultats, la satisfaction du système de santé en Chine augmente avec le fait d'avoir une assurance santé et avec un statut professionnel élevé. Par conséquent, les classes sociales favorisées accèdent plus facilement à des soins de qualité en Chine. Il existe bien une inégalité dans l'accès aux soins de santé des Français résidant en Chine en fonction de leur statut socioéconomique, et cela malgré la mise en place d'une protection sociale chinoise. On peut supposer que la satisfaction des soins reçus par les Français ayant un statut socio-économique élevé est liée à leur fréquentation des cliniques internationales privées.

\section{Les travers du système de santé chinois}

Le système de santé chinois n'a pas évolué assez rapidement face au développement économique pour être en mesure de fournir à tous une couverture sociale de santé. C'est un système à plusieurs vitesses marqué par une opposition villecampagne. Les plus grandes villes proposent des services de santé dont la qua- internationales privées lité les rapproche de ceux engagent des médecins des pays développés, même étrangers, ce qui permet si les coûts sont très éle- de fournir une qualité vés. Ainsi, nous avons essayé des soins avec des standards d'évaluer la qualité des soins internationaux. prodigués en Chine selon la perception des Français résidents ou de passage dans ce pays. L'enquête a été faite auprès d'une population qui a l'habitude de visiter les établissements de santé, puisque $42 \%$ d'entre eux ont consulté au moins quatre fois dans les établissements de santé de Chine (autre que le cabinet médical de l'ambassade de France). Les hôpitaux 


\section{REPÉRAGE}

publics chinois les plus fréquentés à Pékin sont l'hôpital de Chaoyang (24%), I'hôpital Xie he (15\%) et l'hôpital sino-japonais (13 \%). Ces hôpitaux proposent tous des consultations VIP plus adaptées pour les étrangers. Les cliniques internationales privées les plus fréquentées sont Beijing United Family Hospital (45\%), International SOS (39 \%) et l'hôpital Oasis7 (9 \%), parce que plusieurs médecins français y travaillent.

Les patients ont exprimé dans le questionnaire plusieurs raisons de leur insatisfaction vis-à-vis du système de santé en Chine. Les voici énumérées ci-après par ordre de fréquence décroissante. Le coût financier est un problème spécifique aux cliniques internationales pri-

La Chine et l'Inde sont

les plus gros producteurs de médicaments contrefaits dans le monde. Les plus grands

hôpitaux publics chinois

ne sont pas épargnés.

Les cliniques internationales privées s'approvisionnent

auprès des laboratoires pharmaceutiques étrangers implantés en Chine. vées. Le tiers payant n'est pas systématique. Les patients doivent souvent payer leurs frais médicaux puis se les faire rembourser. Nous relevons de nombreuses critiques relatives à la qualité des soins, quel que soit l'établissement de santé, public ou privé. La formation des médecins n'est pas optimale. Les diagnostics de santé ne sont pas précis, établis trop rapidement, voire faux. Les chirurgiens proposent plus souvent qu'en France des interventions chirurgicales ou, au contraire, refusent d'effectuer les actes chirurgicaux faiblement rémunérés avec des risques de complications post-opératoires, comme l'appendicectomie par exemple, par crainte de poursuites judiciaires par le patient. Les médecins prescrivent trop d'antibiotiques, notamment par voie intraveineuse au lieu d'un traitement oral, dans les hôpitaux publics chinois (parce qu'ils ont une obligation de résultats mais aussi pour augmenter leurs revenus trop faibles) ou favorisent la multiplication des examens complémentaires de manière injustifiée dans les cliniques internationales privées. Du fait de ces difficultés, dans le cas des maladies graves ou compliquées, le médecin de l'ambassade de France est souvent amené à proposer au malade un rapatriement en France pour lui assurer une qualité des soins ${ }^{8}$.

L'information médicale donnée au patient par les médecins chinois sur la maladie est insuffisante. Nous avons relevé ce commentaire pour les établissements publics et privés, que ce soit lors des consultations ou lors des hospitalisations. L'éducation thérapeutique des patients atteints notamment d'une maladie chronique est peu explicite. C'est à la fois un problème de temps, les médecins chinois ont trop de consultations, et un problème de formation des médecins, puisque l'enseignement sur l'importance d'une bonne relation médecin-patient reste encore peu développé dans les facultés de médecine en Chine.

La durée d'attente est un problème des hôpitaux publics chinois. Dans les consultations ordinaires, on ne peut pas prendre de rendez-vous. Le patient doit prendre un ticket de consultation le matin et attendre son tour. Les patients sont très nombreux. Dans un hôpital public de taille moyenne en Chine, il y a environ 10 ooo consultations par jour. Il faut prévoir généralement une journée entière pour consulter un médecin. Par contre, dans les hôpitaux publics chinois qui proposent des consultations VIP à un coût plus élevé, le patient peut prendre rendez-vous.

La difficulté de communiquer est un problème majeur des hôpitaux publics chinois. L'ensemble 
du personnel n'a pas une connaissance suffisante de la langue anglaise, encore moins de la langue française. Il est fortement conseillé à un étranger de consulter avec un traducteur, à moins d'avoir un excellent niveau de chinois. Dans les consultations VIP de ces hôpitaux, le personnel est censé parler l'anglais, ce qui est rare en réalité. En revanche, les professionnels de santé dans les cliniques internationales privées consultent en langue anglaise.

L'absence d'une bonne hygiène est une critique qui apparaît fortement dans les questionnaires en ce qui concerne les hôpitaux publics chinois (consultation et hospitalisation). En effet, l'hygiène y est très souvent médiocre. Les locaux ne sont pas suffisamment nettoyés. Les médecins ne respectent pas les règles de désinfection et ne se lavent pas suffisamment les mains. Les draps des patients ne sont pas systématiquement changés. Il n'y a d'ailleurs pas d'aide soignant pour laver les patients. En Chine, la famille a souvent la charge de la toilette du malade hospitalisé et fait ellemême les premiers soins. Les règles d'asepsie dans les blocs opératoires ne sont pas toujours respectées. Les infections nosocomiales restent un sujet tabou. Aucun chiffre n'est déclaré sur ce sujet.

Dans les remarques sur le système de santé en Chine, on note le problème de la sécurité médicamenteuse. La Chine et l'Inde sont les plus gros producteurs de médicaments contrefaits dans le monde. Les plus grands hôpitaux publics chinois ne sont pas épargnés. Les cliniques internationales privées s'approvisionnent auprès des laboratoires pharmaceutiques étrangers implantés en Chine. Cependant, tous les médicaments occidentaux n'y sont pas fabriqués et l'importation est interdite.
En conclusion, il est très compliqué pour un Français de se soigner correctement en Chine, d'autant plus pour celui qui a un statut socio-économique faible puisqu'il n'a pas les moyens de consulter dans les cliniques internationales privées. Sans être optimales, ces cliniques proposent tout de même des soins de meilleure qualité que les hôpitaux publics chinois. C'est dans ce contexte de difficultés d'accès aux soins de santé de qualité que les Français s'adressent au médecin de l'ambassade de France. En effet, 99 \% des personnes ont répondu dans notre enquête que le médecin de l'ambassade était indispensable à Pékin de nos jours. I 